\title{
Correction: Double-blind, placebo-controlled, dose-ranging trial of intravenous ketamine as adjunctive therapy in treatment-resistant depression (TRD)
}

Maurizio Fava ${ }^{1} \cdot$ Marlene P. Freeman $^{1} \cdot$ Martina Flynn $^{1} \cdot$ Heidi Judge $^{1} \cdot$ Bettina B. Hoeppner $^{1} \cdot$ Cristina Cusin $^{1}$. Dawn F. Ionescu ${ }^{1} \cdot$ Sanjay J. Mathew ${ }^{2} \cdot$ Lee C. Chang ${ }^{2} \cdot$ Dan V. Iosifescu $\mathbb{1}^{3} \cdot$ James Murrough $^{3} \cdot$ Charles Debattista $^{4}$. Alan F. Schatzberg ${ }^{4}$ Madhukar H. Trivedi $\mathbb{1}^{5} \cdot$ Manish K. Jha ${ }^{5} \cdot$ Gerard Sanacora $^{6} \cdot$ Samuel T. Wilkinson $^{6}$. George I. Papakostas ${ }^{1}$

Published online: 7 January 2019

(c) Springer Nature Limited 2019

\section{Correction to: Molecular Psychiatry}

https://doi.org/10.1038/s41380-018-0256-5

Supplementary Figure 1 and Supplementary Tables 1-4 have been re-uploaded so as to reflect the versions supplied during proofs stage. The publisher apologizes for the error in versioning. The HTML version of the paper has been updated.

Supplementary information The online version of this article (https:// doi.org/10.1038/s41380-018-0311-2) contains supplementary material, which is available to authorized users.

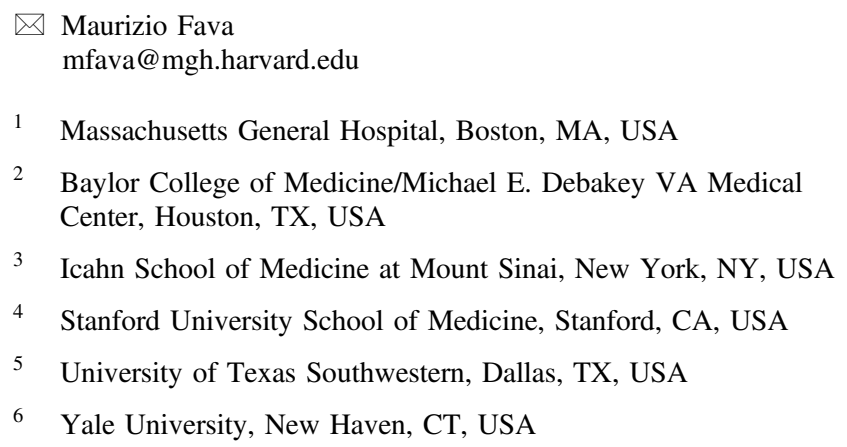

6 Yale University, New Haven, CT, USA 